\title{
Avaliação sensorial de bebidas de goiaba adoçadas com diferentes agentes adoçantes
}

\author{
Sensory evaluation of guava drinks sweetened with different sweetening agents
}

\author{
Aline Gurgel FERNANDES ${ }^{1}$, Paulo Henrique Machado de SOUSA ${ }^{1 *}$, Geraldo Arraes MAIA ${ }^{1}$, \\ Daniele Sales da SILVA ${ }^{1}$, Sandra Maria Lopes dos SANTOS ${ }^{1}$
}

\section{Resumo}

Devido à crescente procura por bebidas à base de frutas tropicais e à expansão do segmento de produtos com valores calóricos reduzidos, este trabalho objetivou avaliar o grau de doçura, sabor, impressão global e a intenção de compra das bebidas de goiaba adoçadas com diferentes agentes adoçantes através de frequência de notas, teste de médias e Mapa de Preferência Interno (MPI). Utilizaram-se polpa de goiaba, água potável e diferentes agentes adoçantes (sacarose, mistura ciclamato/sacarina, aspartame, acesulfame-K, estévia e mistura sacarina/ciclamato sódico/acesulfame-K). A avaliação sensorial foi realizada com 100 provadores não treinados em teste laboratorial através do delineamento de blocos completos balanceados. Objetivando a avaliação das respostas individuais de cada provador, as respostas sensoriais foram avaliadas pela metodologia do MPI, empregando-se a técnica de Análise de Componentes Principais. As bebidas de goiaba adoçadas com sacarose e aspartame apresentaram avaliação sensorial semelhante por parte dos consumidores, apresentando os maiores valores médios no teste de aceitação, enquanto as adoçadas com estévia, com a mistura de três agentes adoçantes e a mistura ciclamato/sacarina apresentaram os valores mais baixos na avaliação sensorial. A utilização do MPI confirmou os resultados obtidos através de frequência de notas e teste de médias.

Palavras-chaves: bebida de goiaba; edulcorantes; mapa de preferência interno.

\begin{abstract}
Due to a constant search for tropical fruit drinks and calorie reduced products, this work aimed to evaluate the degree of sweetness, flavor, overall global impression, and purchase intention of guava drinks sweetened with different sweetening agents through the response rank frequency and average test, and internal preference mapping (IPM). Guava pulp, drinking water, and sweetening agents (sucrose, mixture cyclamate/saccharine, aspartame, acesulfame K, stevia and mixture saccharine/sodium cyclamate/acesulfame K) were used. The sensory evaluation was carried out with 100 untrained tasters in laboratorial tests through the balanced complete block designs. Aiming at the evaluation of the taster's individual responses, the sensorial answers were evaluated through the IPM methodology employing the technique of main component analysis. The guava drinks sweetened with sucrose and aspartame got similar sensorial evaluation by the consumers presenting the highest average values in the acceptance test. Nevertheless, those sweetened with stevia, a mixture of three sweetening agents, and a mixture of cyclamate/saccharine presented the lowest values in the sensory evaluation. The utilization of the IPM confirmed the results obtained using the response rank frequency and average test.

Key words: guava drinks; edulcorants; internal preference map.
\end{abstract}

\section{Introdução}

A goiaba (Psidium guajava L.), dentre as frutas tradicionais, destaca-se como excelente fonte de vitamina $C$, além de possuir quantidades razoáveis de pró-vitamina A e vitaminas do complexo B, minerais como cálcio, fósforo e ferro mostra grande aceitação para o consumo in natura (MAIA et al., 2002; CÁCERES, 1999), tornando-se uma fruta muito importante dentro do contexto da fruticultura brasileira. O aumento do consumo está associado à grande divulgação das qualidades nutricionais da fruta (ZANATTA, 2006). A quantidade produzida em 2005 foi de 345.533 toneladas no Brasil, sendo 156.886 toneladas na região Nordeste, segundo o Instituto Brasileiro de Geografia e Estatística (IBGE, 2007).

Os indivíduos que, por diversas razões, precisam substituir a sacarose por adoçantes não calóricos procuram por produtos que sejam dotados de gosto e características próximas às da

sacarose (CARDELLO; SILVA; DAMÁSIO 1999; CARDOSO; BATTOCHIO; CARDELLO, 2004). Esta constitui a substância mais utilizada como adoçante de bebidas devido às suas características únicas de sabor (VERMUNT et al., 2003). Várias substâncias surgiram para suprir esta necessidade, mas poucas foram comprovadamente estabelecidas como seguras para o consumo humano, com bom poder edulcorante e estabilidade satisfatória (CARDOSO; BATTOCHIO; CARDELLO, 2004).

Os edulcorantes ou adoçantes são produtos químicos de origem sintética ou natural, que têm a propriedade de adoçar um alimento em substituição total ou parcial do açúcar comercial (BOBBIO; BOBBIO, 1995; CÂNDIDO; CAMPOS, 1996; GROBOTH, 1999; MONTIJANO, TOMÁS-BARBERÁN; BORREGO, 1998). O edulcorante ideal deve apresentar perfil de sabor e propriedades funcionais semelhantes às da sacarose.

Recebido para publicação em 27/9/2007

Aceito para publicação em 14/12/2007 (002892)

'Departamento de Tecnologia de Alimentos, Universidade Federal do Ceará - UFC, Av. Mister Hull, 2977, Campus do Pici, CP 12168, CEP 60356-000, Fortaleza - CE, Brasil, E-mail: phenriquemachado@gmail.com

${ }^{*}$ A quem a correspondência deve ser enviada 
Os edulcorantes permitidos para uso em alimentos e bebidas dietéticas são vários, mas cada um possui características específicas de intensidade e persistência do gosto doce e presença ou não de gosto residual. Além disso, tais características podem modificar-se em função de suas concentrações. Esses fatores são determinantes na aceitação, na preferência e na escolha por parte dos consumidores (CARDELLO; SILVA; DAMÁSIO, 2000).

Dentre os edulcorantes naturais, pode-se destacar o esteviosídeo, um glicídio diterpênico extraído das folhas de Stevia rebaudiana Bertoni. O seu poder adoçante é cerca de 150 a 300 vezes maior que o da sacarose, mas apresenta sabor amargo residual. $\mathrm{O}$ esteviosídeo possui grande aplicação na indústria alimentícia devido a sua estabilidade frente ao calor e a uma ampla faixa de $\mathrm{pH}$ (GOTO; CLEMENTE, 1998).

O sucesso da sacarina pode ser atribuído a alguns fatores: preço cerca de 20 vezes menor que a sacarose; não é metabolizado pelo organismo, portanto, não fornece calorias; não afeta os dentes; é estável e tem ampla faixa de aplicações (HIGGINBOTHAM, 1983). O ciclamato possui a vantagem particular de reduzir o gosto amargo residual da sacarina quando associado a ela, é também isento de calorias, uma vez que não é metabolizado (WELLS, 1989). Os edulcorantes sacarina e ciclamato são muito utilizados associados em diferentes proporções, em função do sinergismo proporcionado. No Brasil existem diversos adoçantes de mesa com esta composição, sendo que os mais vendidos possuem a proporção de duas partes de ciclamato para uma de sacarina.

O N-L-a-aspartil-L-fenilalanina-1-metil éster, mais conhecido como aspartame, possui um poder edulcorante cerca de 200 vezes mais doce que a sacarose a 10\% (CARDELLO; SILVA; DAMÁSIO, 1999; HOMLER, 1988). Baldwin e Korschgen (1979) demonstraram, em suas pesquisas, que o uso de aspartame em bebidas, acentua o aroma, especialmente em sucos de frutas cítricas.

Para que estes edulcorantes sejam aplicados com êxito é necessário que, além de sua segurança absoluta, eles apresentem características sensoriais agradáveis, com doçura semelhante à da sacarose. A única forma de se avaliar a aceitação de um edulcorante é pela análise sensorial.

Os adoçantes são seguros se utilizados na dose correta, de forma não exagerada. Como eles possuem um poder de doçura maior que o açúcar, pequena quantidade é o suficiente para dar sabor a alimentos e bebidas.

Muitos estudos sobre as propriedades sensoriais de edulcorantes vêm sendo publicados (AYYA; LAWLESS, 1992; CARDELLO; SILVA; DAMÁSIO, 1999; HARRISON; BERNHARDT, 1984; KETELSEN; KEAY; WIET, 1993; LARSON-POWERS; PANGBORN, 1978; OTT; EDWARDS; PALMER 1991; SHAMIL et al., 1988; SWARTZ, 1980). Na maioria dos estudos, é feita a avaliação dos edulcorantes isoladamente, e suas associações são pouco discutidas. Estudos anteriores foram feitos para se obter as concentrações dos adoçantes a serem utilizadas e suas doçuras equivalentes em sacarose (CAVALLINI; BOLINI, 2005; CARDOSO et al., 2004; CARDELLO; BOTOCHIO; CARDELO, 1999; CARDELLO; SILVA; DAMÁSIO, 2000).

Com a finalidade de analisar os dados afetivos levando-se em consideração a resposta individual de cada consumidor e não somente a média do grupo de consumidores que testaram os produtos, foi desenvolvida a técnica intitulada Mapa de
Preferência que tem sido largamente utilizada por cientistas da área de análise sensorial na Europa, nos Estados Unidos e Austrália (MACFIE, 1990; MARKETO et al., 1994; SCHLICH; MCEWAN, 1992). Esta técnica é frequentemente empregada com o objetivo de identificar grupos de consumidores que respondam uniformemente e que difiram de outros grupos pela idade, sexo, atitude, necessidade, hábitos alimentares e/ou respostas para os atributos do produto (WESTAD; HERSLETH; LEA, 2004). O Mapa de Preferência Interno pode complementar a análise de aceitação de um produto, explicando as preferências dos consumidores, que se tornam assim informações valiosas (CARDELLO; FARIA, 2000).

Considerando-se a crescente procura por bebidas à base de frutas tropicais e a expansão do segmento de produtos com valores calóricos reduzidos, o estudo do comportamento sensorial da bebida de goiaba adoçada com diferentes edulcorantes mostrou-se oportuno. Este trabalho teve por objetivo comparar os métodos sensoriais de avaliação (frequência de notas, teste de médias e mapa de preferência interno das bebidas de goiaba adoçadas com sacarose e diferentes edulcorantes).

\section{Material e métodos}

\subsection{Material}

Utilizou-se polpa de goiaba, água potável, sacarose, mistura ciclamato/sacarina na proporção $2: 1$, aspartame, acesulfame-K e estévia e mistura sacarina/ciclamato sódico/acesulfame-K.

\subsection{Preparo da amostra}

As bebidas de goiaba foram obtidas pela diluição de 17,5\% de polpa em água mineral e adoçado no momento da análise, com $11 \%$ de sacarose e suas concentrações equivalentes para os demais agentes adoçantes, de acordo com os valores apresentados na Tabela 1 .

\subsection{Análise sensorial}

A análise foi realizada com 100 provadores não treinados (STONE; SIDEL, 1993) em teste laboratorial, através do delineamento de blocos completos balanceados (MACFIE et al., 1989) com codificação aleatória de três dígitos. Os testes foram conduzidos em cabines individuais, sob luz branca (luz do dia), e as amostras foram apresentadas monadicamente em duas sessões. Os provadores receberam $30 \mathrm{~mL}$ de cada amostra à temperatura entre $16 \mathrm{e} 18^{\circ} \mathrm{C}$, em taças de vidro. Os provadores avaliaram a impressão global, sabor e a doçura das amostras utilizando escala hedônica estruturada de nove pontos, na qual os

Tabela 1. Concentração dos agentes adoçantes (\%) adicionados às bebidas de goiaba.

\begin{tabular}{lc}
\hline \multicolumn{1}{c}{ Agente adoçante } & Concentração (\%) \\
\hline Sacarose & 11 \\
Aspartame & 0,0555 \\
Acesulfame-K & 0,035 \\
Mistura ciclamato/sacarina & 0,0358 \\
Mistura sacarina/ciclamato sódico/acesulfame-K & 0,03125 \\
Estévia & 0,06 \\
\hline
\end{tabular}


extremos representavam "gostei muitíssimo" (9) e "desgostei muitíssimo" (1) e o centro "nem gostei, nem desgostei" (5) (PERYAM; PILGRIM, 1957).

\subsection{Análise estatística}

As porcentagens dos valores hedônicos de 1 a 4 foram somadas e denominadas de "\% de rejeição", enquanto que as porcentagens dos valores hedônicos de 6 a 9 foram denominadas de "\% de aceitação"; o valor 5 foi considerado como região de indiferença ("nem gostei, nem desgostei”).

Objetivando-se a avaliação das respostas individuais de cada provador, as respostas sensoriais foram avaliadas pela metodologia do Mapa de Preferência Interno, empregando-se a técnica de Análise de Componentes Principais.

Os valores hedônicos também foram submetidos à análise de variância e as médias submetidas ao teste de Tukey ao nível de $5 \%$ de probabilidade.

As respostas sensoriais também foram avaliadas pela metodologia do Mapa de Preferência Interno (MacFIE; THOMSON, 1988). Os dados da avaliação sensorial foram organizados de acordo com CARNEIRO et al. (2003) numa matriz de amostras (em linhas) e consumidores (em colunas), sendo avaliados a partir da matriz de covariâncias. Os resultados foram apresentados em gráficos de dispersão das amostras (tratamentos) e de cada consumidor em relação às duas primeiras dimensões principais.

As análises estatísticas foram realizadas através do programa estatístico SAS (Statistical Analysis System) (2006), versão 9.1.

\section{Resultados e discussão}

A Tabela 2 apresenta a distribuição das notas de acordo com a aceitação, rejeição ou indiferença dos provadores, juntamente com os valores hedônicos médios de cada formulação e teste de Tukey.

Para a doçura, as bebidas de goiaba adoçadas com sacarose e aspartame apresentaram frequência de notas na zona de aceitação semelhantes (81\%), enquanto as amostras adoçadas com estévia e a mistura ciclamato/sacarina, os valores apresentaram as maiores quantidades de notas na zona de rejeição (Tabela 1).

No caso dos atributos sabor e impressão global, verificaram-se comportamentos semelhantes por parte dos provadores, que apresentaram maiores frequências para sacarose, seguidas pela bebida adoçada com aspartame. As amostras com estévia e a mistura ciclamato/sacarina apresentaram as maiores quantidades de notas na zona de rejeição (Tabela 1 ).

As amostras adoçadas com sacarose e aspartame tiveram os maiores valores de aceitação para o atributo doçura, não diferindo entre si para o teste de média, ficando entre os termos hedônicos "gostei ligeiramente" e "gostei moderadamente". Estas amostras diferiram do restante, que apresentaram valores próximos ao termo hedônico "não gostei nem desgostei" para as bebidas adoçadas com acesulfame-K e com a mistura sacarina/ciclamato sódico/acesulfame-K, e ficaram entre os termos hedônicos "desgostei moderadamente" e "desgostei muito" das bebidas adoçadas com ciclamato/sacarina e estévia.
Resultados semelhantes foram observados por Cavallini e Bolini (2005) estudando a comparação da percepção temporal de doçura, amargor e sabor de fruta em suco de manga reconstituído e adoçado com sacarose, mistura ciclamato/sacarina 2: 1, aspartame, sucralose e estévia, e concluíram que o aspartame foi o edulcorante cujo perfil temporal mais se aproximou ao da sacarose, para a maioria dos parâmetros estudados em relação ao estímulo doce, e a estévia apresentou o comportamento mais distinto em relação à sacarose.

Cardello, Silva e Damásio (1999) também obtiveram resultado semelhante quando avaliaram o comportamento temporal para o estímulo doce de soluções aquosas de sacarose, aspartame, mistura ciclamato/sacarina 2: 1 e estévia a 10\% de equivalência de doçura, sendo a solução de aspartame a amostra que mais se aproximou da sacarose, enquanto a estévia diferiu em maior grau da sacarose.

As bebidas adoçadas com sacarose e aspartame não apresentaram diferença significativa entre si para o teste de médias, para o atributo sabor, diferindo das amostras de acesulfame-K e da mistura sacarina/ciclamato sódico/acesulfame-K, que apresentaram valores entre os termos hedônicos "não gostei nem desgostei" e "gostei ligeiramente". As bebidas adoçadas com ciclamato/sacarina e estévia não apresentaram diferença significativa entre si, diferindo do restante e apresentando valores próximos ao termo hedônico "desgostei moderadamente".

Para a impressão global, as amostras de suco adoçadas com sacarose e aspartame não diferiram estatisticamente entre si, ficando com valores entre os termos hedônicos "gostei ligeiramente" e "gostei moderadamente", diferindo das amostras adoçadas com a mistura sacarina/ciclamato sódico/acesulfame-K e acesulfame-K, que ficaram com valores entre os termos hedônicos "não gostei, nem desgostei" e "gostei ligeiramente".

\subsection{Mapa de preferência interno}

O Mapa de Preferência Interno é uma análise de componentes principais (ACP) na matriz de dados, consistindo de amostras ou produtos (objetos) e consumidores (variáveis), do mesmo modo que ACP identifica a maior fonte de variação e extrai estas como componentes. O resultado é um mapa de amostra e um mapa de consumidor, correspondendo, respectivamente, aos escores e às cargas da ACP (HELGENSEN; SOLHEIN; NAES, 1997). O número relativo de consumidores no segmento reflete a variação dentro da categoria do produto, mas esta varia dependendo do tipo de produto (WESTAD; HERSLETH; LEA, 2004).

\section{Atributo sabor}

Com os dados obtidos no teste de aceitação das seis formulações das bebidas de goiaba adoçadas com sacarose e cinco formulações com edulcorantes foram obtidos os Mapas de Preferência Interno do atributo doçura (Figura 2).

O Mapa de Preferência Interno gerou, em espaço multidimensional, as coordenadas relativas ao produto, que por sua vez, foram formadas de acordo com as respostas dos provadores. Os mapas da Figura 2 foram gerados por meio dos 
Componentes Principais (CP-1, CP-2), que juntos explicaram $80,4 \%$ para o atributo sabor e o CP-1 explicou 70,2\% da variação e o CP-2 $10,2 \%$.

Na Figura 1, cada ponto em preto representa as correlações entre os dados de aceitação de um consumidor e os dois primeiros componentes principais. Dessa forma, cada ponto preto está associado a um provador.

As amostras sacarose, mistura sacarina/ciclamato sódico/acesulfame-K e aspartame se agruparam formando um grupo de provadores, enquanto as amostras estévia e

Tabela 2. Resultados da percentagem de rejeição, indiferença e aceitação e teste de Tukey de bebida de goiaba adoçada com sacarose e diferentes edulcorantes.

\begin{tabular}{|c|c|c|c|c|}
\hline \multirow[t]{2}{*}{ Edulcorante } & \multicolumn{3}{|c|}{ Doçura } & \multirow[t]{2}{*}{ Médic } \\
\hline & \% de rejeição & \% de indiferença & \% de aceitação & \\
\hline Sacarose & 15 & 5 & 81 & $6,6^{\mathrm{a}}$ \\
\hline Aspartame & 17 & 3 & 81 & $6,4^{\mathrm{a}}$ \\
\hline Acesulfame-K & 34 & 16 & 61 & $5,5^{\mathrm{b}}$ \\
\hline Ciclamato/Sacarina & 93 & 3 & 5 & $2,4^{\mathrm{c}}$ \\
\hline Sacarina/Ciclamato sódico/Acesulfame-K & 34 & 8 & 59 & $5,6^{\mathrm{b}}$ \\
\hline Estévia & 96 & 0 & 5 & $2,2^{c}$ \\
\hline \multirow[t]{2}{*}{ Edulcorante } & & Sabor & & Média \\
\hline & \% de rejeição & $\%$ de indiferença & \% de aceitação & \\
\hline Sacarose & 14 & 10 & 77 & $6,5^{\mathrm{a}}$ \\
\hline Aspartame & 10 & 4 & 87 & $6,7^{\mathrm{a}}$ \\
\hline Acesulfame-K & 35 & 10 & 56 & $5,4^{\mathrm{b}}$ \\
\hline Ciclamato/Sacarina & 86 & 3 & 12 & $2,8^{\mathrm{c}}$ \\
\hline Sacarina/Ciclamato sódico/Acesulfame-K & 29 & 10 & 62 & $2,7^{c}$ \\
\hline Estévia & 85 & 3 & 13 & $2,9^{c}$ \\
\hline \multirow[t]{2}{*}{ Edulcorante } & & Impressão global & & Média \\
\hline & \% de rejeição & $\%$ de indiferença & \% de aceitação & \\
\hline Sacarose & 14 & 9 & 78 & $6,6^{\mathrm{a}}$ \\
\hline Aspartame & 10 & 5 & 86 & $6,7^{\mathrm{a}}$ \\
\hline Acesulfame-K & 32 & 13 & 56 & $5,5^{\mathrm{b}}$ \\
\hline Ciclamato/Sacarina & 86 & 3 & 12 & $2,8^{\mathrm{c}}$ \\
\hline Sacarina/Ciclamato sódico/Acesulfame-K & 27 & 14 & 60 & $5,8^{\mathrm{b}}$ \\
\hline Estévia & 82 & 6 & 13 & $3,0^{c}$ \\
\hline
\end{tabular}

${ }^{*}$ Médias seguidas da mesma letra não apresentam diferença significativa $(\mathrm{p}>0,05)$ pelo teste de Tukey.

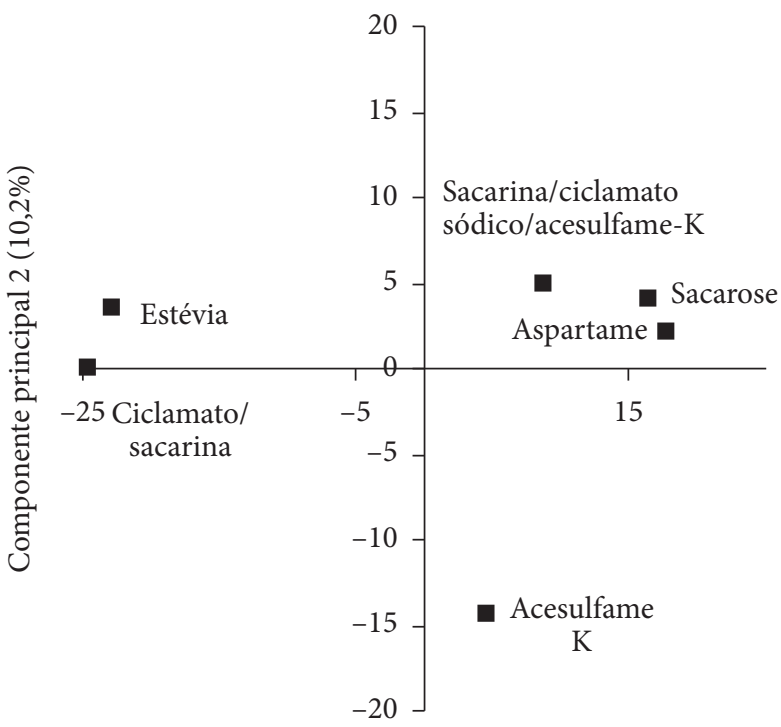

Componente principal $1(70,2 \%)$

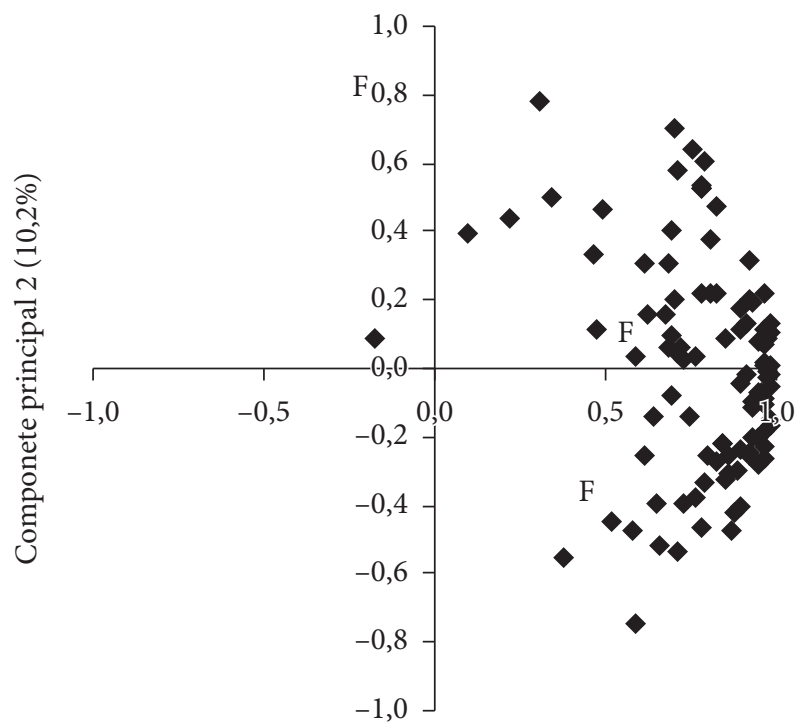

Componente principal $1(70,2 \%)$

Figura 1. Mapa de Preferência Interno, do atributo sabor, para bebida de goiaba adoçada com sacarose, aspartame, mistura sacarina/ciclamato sódico/acesulfame-K, estévia, mistura ciclamato/sacarina e acesulfame-K. 


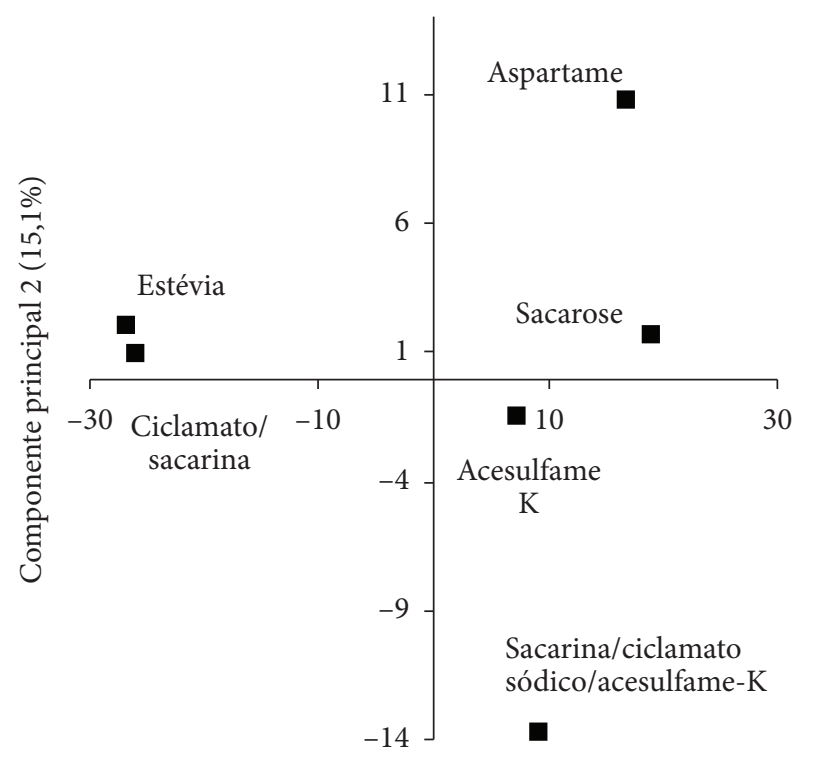

Componente principal $1(70,4 \%)$

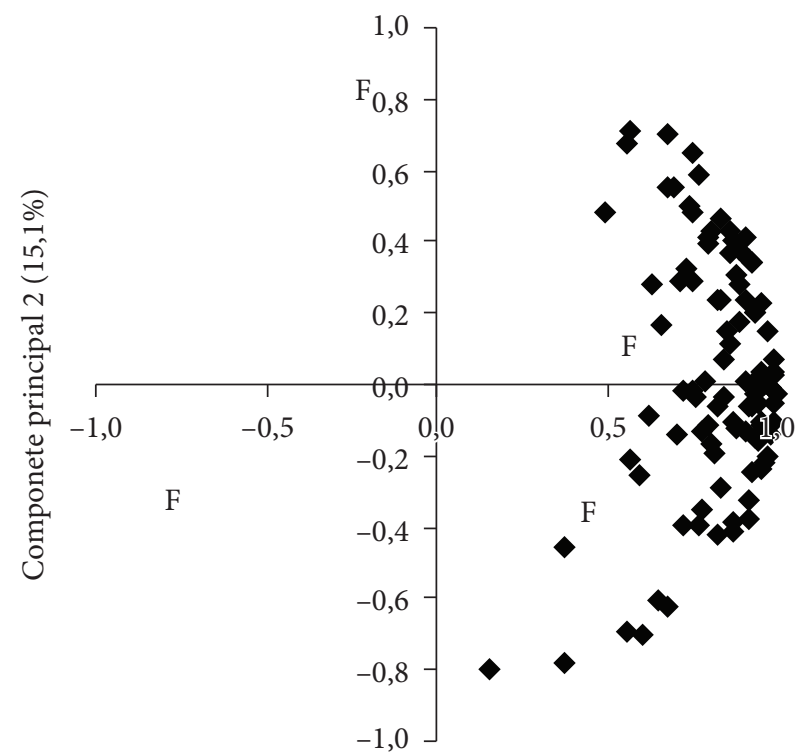

Componente principal $1(70,4 \%)$

Figura 2. Mapa de Preferência Interno, do atributo doçura, para bebida de goiaba adoçada com sacarose, aspartame, mistura sacarina/ciclamato sódico/acesulfame-K, estévia, mistura ciclamato/sacarina e acesulfame-K.

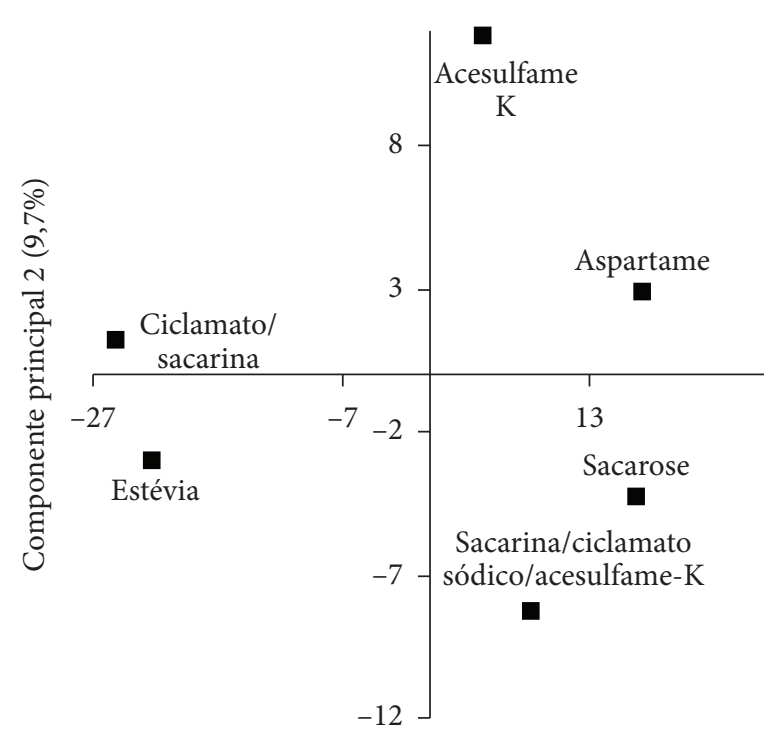

Componente principal $1(70,7 \%)$

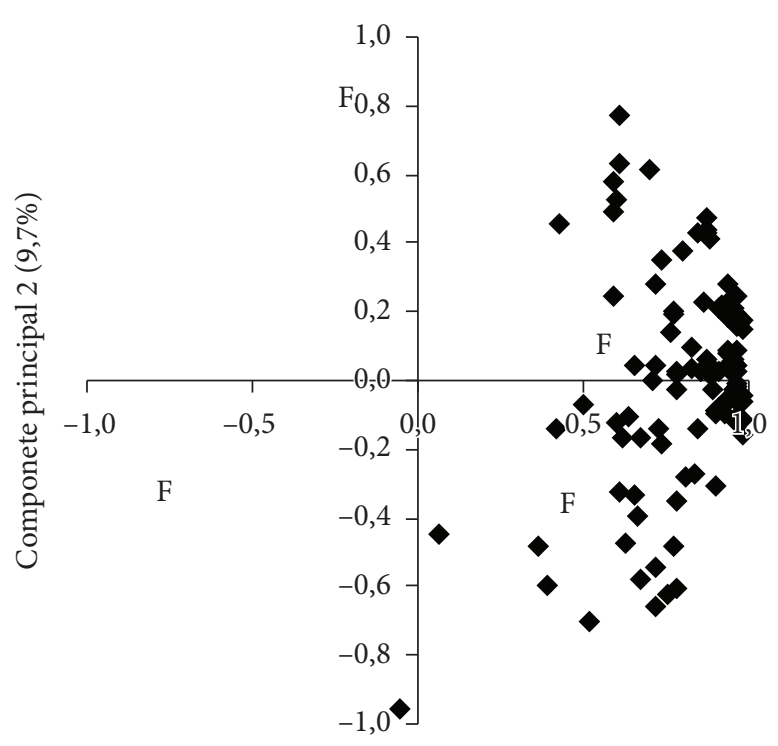

Componente principal $1(70,7 \%)$

Figura 3. Mapa de Preferência Interno, para Impressão global, para bebida de goiaba adoçada com sacarose, aspartame, mistura sacarina/ciclamato sódico/acesulfame-K, estévia, mistura ciclamato/sacarina e acesulfame-K.

ciclamato/sacarina formaram outro grupo. A amostra com acesulfame-K apresentou-se isoladamente, não estando associada à formação de grupo (Figura 1). Não houve provadores localizados no centro do gráfico, sendo que todos discriminaram as amostras em relação ao sabor.

Quanto à correlação entre os dados de aceitação de cada consumidor com os dois primeiros componentes principais, verificou-se uma preferência nítida dos consumidores pelo grupo formado pelas amostras adoçadas com sacarose, mistura sacarina/ciclamato sódico/acesulfame-K e aspartame. Não foram observados consumidores próximos às amostras com adição da estévia e da mistura ciclamato/sacarina. Já a amostra adoçada com acesulfame-K teve alguns consumidores próximos a ela (Figura 1).

\section{Atributo doçura}

Os dois primeiros (CP-1 e CP-2) juntos explicaram 85,5\% para o atributo doçura. Não foram observados consumidores 
alocados no centro do gráfico em relação ao atributo sabor. Não foi verificada uma nítida distinção na formação de grupos em relação ao atributo sabor para as bebidas de goiaba adicionadas de sacarose e edulcorantes, exceto para as amostras adicionadas de estévia e da mistura ciclamato/sacarina. Entretanto, verificou-se uma proximidade maior dos consumidores às amostras adoçadas com sacarose e acesulfame- $K$; enquanto as amostras com aspartame e a mistura sacarina/ciclamato sódico/acesulfame-K tiveram menores quantidades de consumidores próximos a elas (Figura 2).

As amostras adoçadas com sacarose e acesulfame- $\mathrm{K}$ apresentaram preferência por um maior número de provadores, situando-se à direita do gráfico; enquanto as amostras com estévia e a mistura ciclamato/sacarina apresentaram-se à esquerda do gráfico, não tendo a preferência dos provadores em relação ao sabor.

\section{Impressão global}

Os mapas da Figura 3 explicaram 80,4\% de variação para a impressão global. Nesta não foi observada a formação de grupos. Houve uma maior concentração de provadores no lado direito dos gráficos (consumidores representados no gráfico por pontos pretos), próximos às amostras das bebidas adicionadas de sacarose e aspartame, havendo uma aceitação homogênea, uma vez que não houve definição de preferência dos consumidores em relação a uma amostra específica.

\section{Conclusões}

As bebidas de goiaba adoçadas com sacarose e aspartame apresentaram avaliação sensorial semelhante por parte dos consumidores, apresentando os maiores valores médios no teste de aceitação, enquanto os adoçados com a mistura sacarina/ciclamato sódico/acesulfame-K, estévia e a mistura ciclamato/sacarina apresentaram os valores mais baixos na avaliação sensorial.

A utilização do Mapa de Preferência Interno através de Análise de Componentes Principais confirmou os resultados obtidos através de frequência de notas e teste de médias.

\section{Agradecimentos}

À Fundação Cearense de Apoio ao Desenvolvimento Científico e Tecnológico (FUNCAP) e ao CNPq pela concessão de bolsas.

\section{Referências bibliográficas}

AYYA, N.; LAWLESS, H. T. Quantitative and qualitative evaluation of high-intensity sweeteners and sweetener mixtures. Chemical Senses, v. 17, n. 3, p. 245-259, 1992.

BALDWIN, R. E.; KORSCHGEN, B. M. Intensification of fruit flavors by aspartame. Journal of Food Science, v. 44, n. 3, p. 938-939, 1979.

BOBBIO, P. A.; BOBBIO, F. O. Química do processamento de alimentos. São Paulo: Ed. Varela, 1995. p. 121-135.

CÁCERES, A. Plantas de uso medicinal em Guatemala. [S.L.]: Editorial Universitária, 1999. 402 p.
CÂNDIDO, L. M. B.; CAMPOS, A. M. Alimentos para fins especiais: dietéticos. São Paulo: Livraria Varela, 1996.

CARDELLO, H. M. A. B.; FARIA, J. B. Análise da aceitação de aguardentes de cana por testes afetivos e mapa de preferência interno. Ciência $\mathbf{e}$ Tecnologia de Alimentos, v. 20, n. 1, p. 32-36, 2000.

CARDELLO, H. M. A. B.; SILVA, M. A. A. P.; DAMÁSIO, M. H. Análise descritiva quantitativa de edulcorantes em diferentes concentrações. Ciência e Tecnologia de Alimentos, v. 20, n. 3, p. 318-328, 2000.

CARDELlO, H. M. A. B.; SILVA, M. A. A. P.; DAMÁSIO, M. H. Análise tempo-intensidade dos estímulos doce e amargo de extrato de folhas de estévia [Stevia rebauduiana (Bert.) Bertoni] em doçura equivalente a sacarose. Ciência e Tecnologia de Alimentos, v. 19, n. 2, p. 163-169, 1999.

CARDOSO, J. M. P.; BATTOCHIO, J. R.; CARDELLO, H. A. B. Equivalência de dulçor e poder edulcorante de edulcorantes em função da temperatura de consumo em bebidas preparadas com chá-mate em pó solúvel. Ciência e Tecnologia de Alimentos, v. 24, n. 3, p. 448-452, 2004.

CARNEIRO, J. D. S. et al. Avaliação sensorial e mapa de preferência interno de marcas comerciais de refrigerante sabor guaraná. Boletim do CEPPA, v. 21, n. 2, p. 279-292, 2003.

CAVALlinI, D. C. U.; BOLINI, H. M. A. Comparação da percepção temporal de doçura, amargor e sabor de fruta em suco de manga reconstituído e adoçado com sacarose, mistura ciclamato/sacarina 2:1, aspartame, sucralose e estévia. Boletim do CEPPA, v. 23, n. 2, p. 361-382, 2005.

GOTO, A.; CLEMENTE, E. Influência do Rebaudiosídeo A na Solubilidade e no Sabor do Esteviosídeo. Ciência e Tecnologia de Alimentos, v. 18, n. 1, p. 3-6, 1998.

GROBOTH, G. Quality assurance in testing laboratories. Journal of Thermal Analysis and Calorimetry, v. 56, n. 3, p. 1405-1412, 1999.

HARRISON, S. K.; BERNHARDT, R. A. Time-intensity sensory characteristics of saccharin, xylitol and galactose, and their effect on the sweetness of lactose. Journal of Food Science, v. 49, n. 3, p. 780-793, 1984.

HELGENSEN, H.; SOLHEIN, R.; NAES, T. Consumer preference mapping of dry fermented lamb sausages. Food Quality and Preference, v. 8, n. 2, p. 97-109, 1997.

HIGGINBOTHAM, J. D. Recent developments in non-nutritive sweeteners. In: GREMBY, T. H.; PARKER, K. J.; LINDLEY, M. G. (Eds.). Developments in sweeteners-2. London: Applied Science. Publ., 1983. p. 119-55.

HOMLER, B. Nutrasweet biond sweetener: a look beyond the taste. In: BIRCH, G. G.; LINDLEY, M. G. (Eds.). Low calorie products. London: Elsevier Applied Science, 1988. p. 113-25.

INSTITUTO BRASILEIRO DE GEOGRAFIA E ESTATÍSTICA - IBGE. Produção Agrícola Municipal. Rio de Janeiro, 2006. Disponível em: <http://www.sidra.ibge.gov.br>. Acesso em: 27 de Fevereiro de 2007.

KETELSEN, S. M.; KEAY, C. L.; WIET, S. G. Time-intensity parameters of selected carbohydrate and high potency sweeteners. Journal of Food Science, v. 58, n. 6, p. 1418-7421, 1993.

LARSON-POWERS, M.; PANGBORN, R. M. Descriptive analysis of the sensory properties of beverages and gelatins containing sucrose or synthetic sweeteners. Journal of Food Science, v. 43, n. 1, p. 47-51, 1978.

MACFIE, H. J. H. Assessment of the sensory properties of food. Nutrition Reviews, v. 48, n. 2, p. 87-93, 1990. 
MACFIE, H. J. H.; THOMSON, D. M. H. Preference Mapping and Multidimensional Scaling. In: PIGGOT J. R. (Ed.). Sensory Analysis of Foods. London: Elsevier, 1988. 389 p.

MACFIE, H. J.; N., BRATCHELL; GREENHOFF, K.; VALLIS, L. Designs to balance the effect of order of presentation and first-order carry-over effects in hall tests. Journal of Sensory Studies, v. 4, n. 2, 129-148, 1989.

MAIA, G. A. et al. Técnica aumenta tempo de conservação da goiaba. Revista Ciência e Tecnologia - FUNCAP, v. 4, n. 1, p. 11-12, 2002.

MARKETO, C. G.; COOPER, T.; PETTY, M. F.; SCRIVEN, F. M. The Reliability of MDPREF to Show Individual Preference. Journal of Sensory Studies, v. 9, n. 3, p. 337-350, 1994.

MONTIJANO, H.; TOMÁS-BARBERÁN, F. A.; BORREGO, F. Propriedades tecnológicas y regulación de los edulcorantes de alta intensidade en la Unión Europea. Food Science and Technology International, v. 4, n. 1, p. 5-16, 1998.

OTT, D. B., EDWARDS, C. L., PALMER, S. J. Perceived taste intensity and duration of nutritive and non-nutritive sweeteners in water using time-intensity (T-I) evaluations. Journal of Food Science, v. 56, n. 2, p. 535-542, 1991.

PERYAM, D. R.; PILGRIM, P. J. Hedonic scale method for measuring food preferences. Food Technology, v. 11, n. 9, p. 9-14, 1957.

SAS INSTITUTE, Inc. SAS User's Guide: version 9.1. Cary: SAS Institute, 2006
SCHLICH, P.; MCEWAN, J. A. Preference mapping: a statistical tool for the food industry. Science des Aliments, v. 12, n. 3, p. 339-355, 1992.

SHAMIL, S. et al. Use of intensity-time studies as an aid to interpreting sweet taste chemoreception. Chemical Senses, v. 13, n. 4, p. 204-215, 1988.

STONE, H. S.; SIDEL, J. L. Sensory Evaluation Practices. San Diego: Academic Press, 1993. 308p.

SWARTZ, M. Sensory screening of synthetic sweeteners using time-intensity evaluations. Journal of Food Science, v. 45, n. 3, p. 577-581, 1980.

VERMUNT, S. H. F.; PASMAN, W. J.; SCHAAFSMA; G.; KARDINAAL, A. F. M. Effects of sugar intake on body weight: a review. Obesity Reviews, v. 2, n. 4, p. 91-99, 2003.

WELLS, A. G. The use of intense sweeteners in soft drinks. In: GREMBY, T. H. (Ed.). Progress in sweeteners. New York: Elsevier Applied Science, 1989. p. 121-42.

WESTAD, F.; HERSLETH, M.; LEA, P. Strategies for consumer segmentation with applications on preference data. Food Quality and Preference, v. 15, n. 7-8, p. 681-687, 2004.

ZANATTA, C. L.; ZOTARELLI, M. F.; CLEMENTE, E. Peroxidase (POD) e polifenoloxidase (PPO) em polpa de goiaba (Psidium guajava $\mathrm{R}$.). Ciência e Tecnologia de Alimentos, v. 26, n. 3, p. 705-708, 2006. 